\title{
A BRIEF DESCRIPTION ON OPTIMIZATION TECHNIQUES
}

\author{
Madhav Singh Solanki \\ SOEIT, Sanskriti University, Mathura, Uttar Pradesh, India
}

\begin{tabular}{|c|c|c|c|}
\hline Journal & \multicolumn{3}{|c|}{$\begin{array}{l}\text { Samvakti Journal of Research in Information Technology } \\
\text { https://www.sjrit.samvaktijournals.com } \\
\text { Volume } 2 \text { Year of Volume } 2021 \text { Page No : } 125-132\end{array}$} \\
\hline Discipline & \multicolumn{3}{|c|}{ Optimization and Scaling } \\
\hline Conference & \multicolumn{3}{|c|}{$\begin{array}{l}\text { A virtual international conference on redefining and transforming the role of higher } \\
\text { education in sustainable development }\end{array}$} \\
\hline Conference & \multicolumn{3}{|c|}{ Start Date: September 30, 2021} \\
\hline Dates & \multicolumn{3}{|c|}{ End Date : September 30, 2021} \\
\hline Institute Name & \multicolumn{3}{|c|}{$\begin{array}{l}\text { JAIN (Deemed-to-be University) in association with Council for Industrial } \\
\text { Innovation and Research }\end{array}$} \\
\hline $\begin{array}{l}\text { Date Received } \\
\text { ID } \\
\text { Dol }\end{array}$ & $\begin{array}{l}\text { November } 21,2021 \\
2021.02 .25 \\
10.46402 / 2021.02 .25\end{array}$ & $\begin{array}{l}\text { Publication Date } \\
\text { Paper Type } \\
\text { Dol URL }\end{array}$ & $\begin{array}{l}\text { : December } 11,2021 \\
\text { : Conference Paper } \\
: \text { https://dx.doi.org/10.46402/2021.02.25 }\end{array}$ \\
\hline
\end{tabular}

\section{ABSTRACT}

The optimization of large-scale issues is fraught with challenges. Multi-modality, dimensionality, and differentiability are the main challenges. Traditional methods often fail to tackle such large-scale issues, particularly when the goal functions are nonlinear. The primary issue is that conventional methods cannot handle non-differentiable functions since most traditional techniques need gradient information, which is not available. Furthermore, such methods often fail to handle optimization problems with a large number of local optima. To address these issues, stronger optimization methods must be developed. Modern optimization techniques are the name given to these methods. This article discusses optimization issue formulation, optimization methodologies, and solution approaches. Methods based on population are also discussed. For structures with discrete parameters, optimization utilizing constraints in terms of dependability is shown to be the optimal choice.

KEYWORDS: Evolutionary Approaches, Genetic Procedures, Memetic Algorithms, Particle Swarm Optimizations. 


\section{INTRODUCTION}

The concept of deciding on the optimal method to complete a job with certain criteria dates back to the dawn of civilisation. The technique of getting the optimal policies to meet specific goals while still meeting specified criteria is known as optimization. Many complex engineering problems may now be effectively optimized thanks to recent developments in the applied mathematics, operational examination, as well as digital computer technologies, and optimization method has therefore gained the status of a science quite than an art.

The majority of engineering need a long design process. Engineers would try out a variety of experimental designs to identify the best one. Engineering design difficulties may be framed as optimization problems with the objective of decreasing cost or weight while still achieving all design constraints.

The recent development of multidisciplinary design optimization (MDO) was prompted by the success of structural optimization in previous decades. Multi-disciplinary designs optimizations is a branch of the engineering that combines a variety of disciplines to address structural design issues using optimization techniques. It is feasible to combine all of the necessary disciplines at the same time. Such issues have a better optimum than sequential optimization of each field.

\section{Optimum Problem Formulation:}

\section{a. Design of variables:}

The design factors are the parameters used to determine the design of a system, and they are mostly updated throughout the optimization process. The first step in developing an optimization problem is to identify the design variables. Since one parameter may be more relevant in terms of decreasing design cost while being inconsequential in terms of optimizing product life, there is no rigorous rule for identifying the variables that may be significant in a situation a priori. As a result, the user's choice of key parameters in an optimization issue is heavily influenced. Cross sectional dimensions, thickness, and other design factors are often used. Bounded variables, or those with a maximum and lowest value, are common in design. These limits may be regarded as restrictions depending on the solution technique ${ }^{[1][2]}$.

\section{b. Constraints:}

The constraints are functional connections between design variables and other design factors that meet specific physical phenomena and resource restrictions. There are three kinds of constraints: 


\section{i. Constraints on inequity:}

When the functional connections among design variables are higher than, lower than, or equal to a resource value, inequality restrictions occur. As a consequence, these constraints guarantee that no failure mode exists or that the behavior is acceptable under the specified loading circumstances.

\section{ii. Constraints on equality}

The criteria that must perfectly match the resources values in condition for the design to be accepted are known as equality constraints.

\section{iii. Constraints on the side}

The maximum and lowest values of design variables are examples of side constraints. Geometric restrictions are applied to the design variables.

\section{c. Objective Function:}

There will be multiple possible solutions for most engineering designs, and the purpose of optimization is to identify the best one among them. As a consequence, criteria for selecting the best choice from among the available possibilities must be created. The criterion must be a scalar function of the model parameters that has a numerical value that can be determined once the design is specified. The optimization problem's objective function is a set of criteria like this.

\section{d. Methods of Optimization:}

There are a variety of optimization techniques that may be utilized to effectively find the optimal values. There are two types of search techniques: gradient and direct search methods.

\section{Gradient based methods:}

To discover the optimum solution, gradient-based approaches employ mathematics as well as derivatives of the objective function, as well as restrictions. In general, it is assumed that optimization algorithms that need gradient information are more efficient. During the design variable optimization process, the restrictions imposed on the solution must also be taken into account. There are many methods for selecting the optimum combination of design variables that will result in the lowest or highest value for a given function. There are two fundamental techniques to obtain the optimal value in the design field: using a differential method or using a search method. Issues with constraints and problems without constraints are two types of problems that may be solved using these two strategies. For problems with no restrictions, the differential calculus technique is 
regarded to be the best way to arrive to an optimum solution. When dealing with constraints, you have two options: differential calculus approaches or search

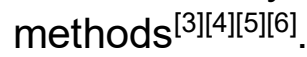

\section{Non - gradient based methods:}

Non-gradient techniques are those that execute a search utilizing merely function values at various places rather than the partial derivatives of the function. Simple issues with a limited number of variables are best solved using these strategies.

\section{Multi-disciplinary Design Optimizations Procedures:}

\section{a. Methodology Response surface:}

RSM is a technique for creating approximation models based on physical investigations or computer simulations. The objective is to enhance a response (output variable) that is impacted by a variety of input factors. Response Surfaces Methodology (RSM) is a collection of statistical as well as mathematical tools for creating, enhancing, and optimizing processes.

\section{b. Evolutionary methods:}

Xie and Steven developed the Evolutionary Structural Optimization (ESO) method in 1992, which is a new structural optimization method. Observations of natural processes and evolution led to the development of this method. Both local and global minima may be found using ESO. Because of its evolutionary features, it does not stop searching for a better one when an apparent minimum is found. The concept behind this strategy was to gradually remove everything under material from of the structural domain in an evolutionary way. The resulting structure will be lighter and have a unique shape. The basic purpose of ESO is to determine the optimum shape and topology for a structure.

\section{c. Problem Solution:}

To solve the problem, the most suitable techniques from the arena of optimizations should be chosen. Algorithms based on gradients, population-based algorithms, and others are among them.

\section{i. Gradient-based methods:}

- Steepest descent technique:

Start with an approximate estimate for the most basic design. The sharpest drop direction is then computed at that location. If the direction is not zero, a line search along the negative of the derivative direction is used to find the smallest point in that direction. The 
search proceeds from the lowest point, which becomes the current position. Continue till you reach convergence.

\section{- Newton's method:}

To generate search directions, this method employs second order derivatives. Second order Taylor's series expansion is used to determine the function value around the current point. Any design iteration that results in a positive semi fixed Hessian matrix yields a global smallest.

\section{- Conjugate gradient methods:}

It's a tweaked type of the steepest descents technique. The recursive equation can be used to find the search directions. After a few rounds, the search direction achieved becomes linearly dependent. The amount of linear dependency is determined by calculating the included angle between two successive search directions. The procedure must be redone if the included angle is extremely near to zero.

\section{ii. Direct search methods:}

- Hooke- Jeeves methods:

It is a sequential strategy in which each step is made up of two sorts of moves: exploration and pattern. The exploratory move takes place in the immediate vicinity of the current site in attempt to find the optimum spot nearby. The pattern search is then performed using two such locations.

\section{- Method of Powell's conjugate direction:}

Powell's technique is the greatest frequently used form of direct search. It creates new search paths based on the history of prior answers. A collection of linearly self-governing directions is generated, as well as uni-directional searches are conducted beside each of these directions, starting at the preceding best position.

\section{Method based Population based:}

\section{a. Genetic algorithms:}

John $\mathrm{H}$. a lecturer at the University of Michigan, generated genetics algorithms. Natural selection and natural genetics are used to create genetic algorithms, which are computerized search and optimization algorithms. They combine survival of the species among string patterns with a systematic information exchange controlled by random operators to generate an acceptable search mechanism. The population of string structures used by genetic algorithms is produced at random. Following that, each string is evaluated. To develop a better population, three operators - reproduction, cross-over, 
and mutation - are used to regulate the random population. The reproduction operator creates the mating pool by selecting good strings from a population. The third operation mutation changes the string locally to generate a better string with a small chance. After then, the population is evaluated and studied to determine whether it should be eliminated. The population is operated periodically by these operators until the termination requirements are reached.

\section{b. Particle swarm optimizations:}

PSO is a stochastic optimization algorithm approach inspired by flocking birds and schooling fish behavior. To produce new populations, no natural evolution-inspired operations are used. The exchange of information between particles, or individuals, in a population, or swarm, is the basis of PSO. Each swarm particle is seen as a point in hyperspace, moving through it with two important abilities: memory of their optimal position (local best) and awareness about their worldwide or neighborhood's best (global best). The basic notion is for each particle to accelerate toward its best local and global performance. The fitness function is being used to evaluate the performance of each particle. Convergence is affected by the inertia weight. The inertia weight is used to control how the velocity of the past effects the velocity of the present. To create a balance between both the swarm's local and global exploration capacities, an adequate inertia weight value should be used, resulting in the best solution.

\section{DISCussion}

Optimization is a process that is used everywhere, from design process to financial markets, from fashion technology to mass communication, and even in our daily lives. We're always trying to improve or improve something called the goal function. In identifying the optimal solution for objective functions, many optimization approaches such as linear regression, simplex method, assignment models, transportation model, CPM, and PERT are significant. Several optimization as well as solution strategies are discussed in this article.

\section{CONCLUSION}

This chapter discusses several optimization and solution techniques. When the goal function's gradient is very close to zero, most traditional approaches fail. They do not ensure that worldwide optimum solutions will be found. To assess the objective function in most actual engineering situations, simulations are needed. As a result, in certain cases, a precise assessment of the gradient may be impossible. This implies that robust search methods, such as the genetic algorithm, are needed to tackle discrete or discontinuous issues. They use a variable coding system to make the search space discrete, despite the fact that the function is continuous. They process many strings at 
the same time, and the result is a global optimum. Probabilistic principles may also be used to guide the search of genetic algorithms. 


\section{REFERENCES:}

[1] A. Mostafaie, E. Forootan, A. Safari, and M. Schumacher, "Comparing multiobjective optimization techniques to calibrate a conceptual hydrological model using in situ runoff and daily GRACE data," Comput. Geosci., 2018, doi: 10.1007/s10596018-9726-8.

[2] S. Sinha and S. S. Chandel, "Review of recent trends in optimization techniques for solar photovoltaic-wind based hybrid energy systems," Renewable and Sustainable Energy Reviews. 2015, doi: 10.1016/j.rser.2015.05.040.

[3] A. Das and B. Datta, "Application of optimisation techniques in groundwater quantity and quality management," Sadhana - Acad. Proc. Eng. Sci., 2001, doi: 10.1007/BF02703402.

[4] S. Singh, M. Singh, and S. C. Kaushik, "A review on optimization techniques for sizing of solar-wind hybrid energy systems," International Journal of Green Energy. 2016, doi: 10.1080/15435075.2016.1207079.

[5] S. S. Fayaed, A. El-Shafie, and O. Jaafar, "Reservoir-system simulation and optimization techniques," Stochastic Environmental Research and Risk Assessment. 2013, doi: 10.1007/s00477-013-0711-4.

[6] C. Suchetha and J. Ramprabhakar, "Optimization techniques for operation and control of microgrids - Review," J. Green Eng., 2018, doi: 10.13052/jge19044720.847 . 